\title{
Prefrontal and Medial Temporal Lobe Activity at Encoding Predicts Temporal Context Memory
}

\author{
Lucas J. Jenkins and Charan Ranganath \\ Center for Neuroscience and Department of Psychology, University of California, Davis, California 95616
}

One of the defining features of episodic long-term memory is that it includes information about the temporal context in which an event occurred. Little is known about the regions that support the encoding of temporal information in the human brain, although previous work has suggested a role for the lateral prefrontal cortex (PFC) and medial temporal lobes (MTL). Here we used event-related fMRI to examine the relationship between activity at encoding and subsequent memory for temporal context. Participants were scanned while performing a serial order working memory task with pictures of common objects and were later tested for temporal memory at two different timescales. In the coarse temporal memory test, participants viewed one object from each trial and indicated approximately when during the course of the experiment it had appeared. In the fine temporal memory test, participants were shown the remaining objects from each trial and asked to recall the order in which they had been originally presented. Activity in the parahippocampal cortex predicted subsequent fine temporal accuracy, whereas coarse temporal accuracy was predicted by activity in several regions of the PFC, as well as in the hippocampus. Additional multivoxel pattern analyses revealed evidence implicating the rostrolateral PFC in the representation of time-varying contextual states in a manner similar to that proposed by computational theories of temporal context memory. These results highlight MTL and PFC contributions to temporal memory at the time of encoding and suggest a particular role for the rostrolateral PFC in encoding coarse temporal context.

\section{Introduction}

Episodic memories contain information not only about the content of an event, but about the temporal context in which the event occurred (Tulving, 1972, 1993). This may include information at a very fine level of temporal detail (e.g., the order of words in a list) but may also include broader, more general temporal context information (e.g., the approximate time when the list was encountered). Although temporal memory has been well studied in the behavioral literature (for review, see Friedman, 1993; Marshuetz, 2005), the neural mechanisms responsible for encoding and representing temporal information at any timescale are poorly understood, especially in the human brain.

Available evidence implicates the prefrontal cortex (PFC) and medial temporal lobes (MTL) as regions critical to temporal context memory. For instance, patients with lesions to the PFC are often impaired at tasks involving memory for the order in which items are presented, even though item recognition memory is relatively well preserved (Milner, 1971; Milner et al., 1985, 1991; Shimamura et al., 1990; Kesner et al., 1994; Mangels, 1997). Lesion and physiology studies in rodents suggest that the hippocampus may also be critical for sequence learning (Kesner and Novak, 1982; Agster et al., 2002; Fortin et al., 2002), and recent ensemble recordings from rat hippocampal neurons suggest that

Received March 14, 2010; revised Sept. 16, 2010; accepted Sept. 20, 2010.

This work was supported by National Institutes of Health Grant R01 MH068721.

Correspondence should be addressed to Lucas J. Jenkins, University of California at Davis, Center for Neuroscience, 1544 Newton Court, Davis, CA 95616. E-mail: ljjenkins@ucdavis.edu.

DOI:10.1523/JNEUROSCI.1337-10.2010

Copyright $\odot 2010$ the authors $\quad 0270-6474 / 10 / 3015558-08 \$ 15.00 / 0$ gradual changes in firing patterns over time may underlie memory for the order of specific events (Manns et al., 2007). Human neuroimaging studies have found evidence for both PFC (EylerZorilla et al., 1996; Cabeza et al., 1997, 2002; Konishi et al., 2002; Suzuki et al., 2002; Dobbins et al., 2003; Amiez and Petrides, 2007; St. Jacques et al., 2008; Kimura et al., 2010) and MTL (St. Jacques et al., 2008; Lehn et al., 2009; Tubridy and Davachi, 2010) involvement in long-term temporal-order memory, but with the exception of Tubridy and Davachi (2010), these studies focused exclusively on activity during retrieval.

Here, we used event-related fMRI to assess the relative roles of the PFC, MTL, and other regions in the long-term encoding of temporal information. We also sought to determine whether the mechanisms involved in encoding fine temporal sequences might differ from those that support memory for temporal context across broader timescales. Participants were scanned while performing a working memory task that required them to maintain the order of four objects over a delay. After scanning, memory for the order of the presented objects was assessed at two different timescales. In the fine temporal memory test, participants were asked to recall the order of objects within each working memory trial. In the coarse temporal memory test, participants were asked to remember the order of items across trials. We then examined activity during each trial as a function of subsequent memory performance on the fine and coarse temporal memory tests. In light of theoretical models stressing a gradually evolving contextual representation as the basis for temporal order memory (Howard and Kahana, 2002), we also investigated whether trialto-trial changes in multivoxel activation patterns would predict successful encoding of temporal context. 
A

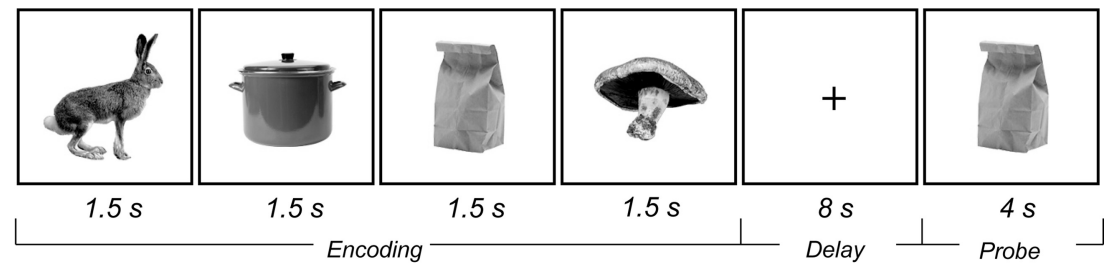

$\mathrm{B}$

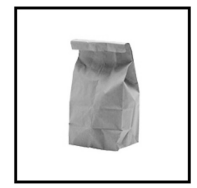

$\mathrm{C}$

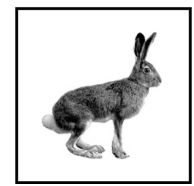

when this item appeared.

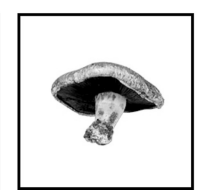

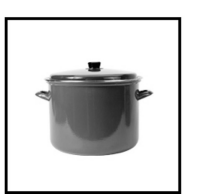$$
\text { origin }
$$
originally appeared.
Figure 1. A, Sample trial sequence. Four objects were presented for $1.5 \mathrm{~s}$ each. A $4 \mathrm{~s}$ probe appeared after an $8 \mathrm{~s}$ delay period at which time participants indicated the serial position of the probed object. $\boldsymbol{B}$, Sample coarse temporal memory test item. Participants were shown one object from the trial and asked to mark on the line approximately when in the experiment it had appeared. C, Sample fine temporal memory test item. Subjects were shown the remaining three objects from the trial and asked to recall the original order of presentation.
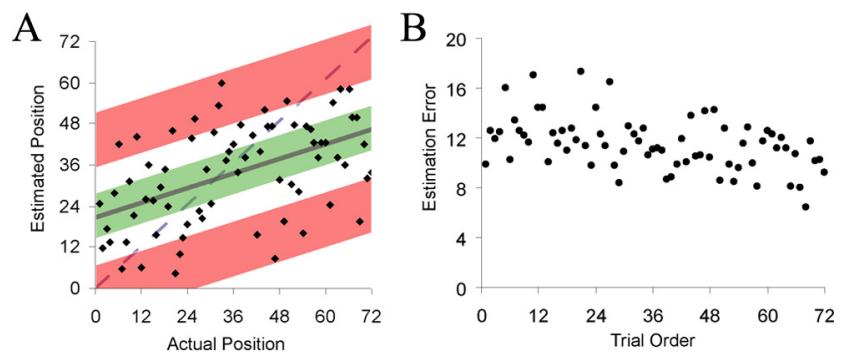

Figure 2. A, Coarse temporal posttest data for a representative participant. The estimated temporal position of each trial is plotted against the order in which the trials originally appeared. The third of trials estimated most accurately (green) were binned as Hits; the third of trials estimated least accurately (red) were binned as Misses. B, Group-averaged residual error for each trial plotted against order of presentation.

\section{Materials and Methods}

Participants. Sixteen healthy, right-handed volunteers (eight females; mean age $=23.06$ years; $\mathrm{SD}=2.82$ ) were recruited from the University of California at Davis student population and paid for their participation. All participants gave informed written consent before scanning. Data from one participant was discarded due to excessive movement artifacts.

Experimental procedure. Stimuli consisted of 288 color photographs of everyday objects, which participants viewed in the context of a serial order working memory task. Each trial began with an encoding period, in which four objects were presented sequentially for $1.5 \mathrm{~s}$ each (Fig. $1 \mathrm{~A}$ ). After an $8 \mathrm{~s}$ delay, a probe object from the previous set appeared for $4 \mathrm{~s}$, and participants were instructed to press one of four buttons on a response box indicating its correct serial position. Trials were separated by a randomly jittered intertrial interval of 6,9 , or $12 \mathrm{~s}$. A total of 72 trials were presented across six 6.3 min scanning runs, for a total functional scanning time of $\sim 45 \mathrm{~min}$.

Two surprise memory tests were administered out of the scanner, $\sim 20$ min after the end of the functional runs. In the coarse temporal memory test, which was given first, participants were shown the probed object from each trial of the working memory task on a computer screen and were instructed to mark on a blank horizontal line approximately when in the experiment the object appeared (Fig. $1 B$ ). They were encouraged to use the full length of the scale, with the left endpoint representing the beginning of the encoding task and the right endpoint representing the end. To minimize the likelihood of item memory, as opposed to temporal order memory, influencing the results, participants were instructed to respond only if they recognized the object from the study phase.

In the fine temporal memory test, which was administered after completion of the coarse temporal memory test, participants were shown the remaining three objects from each trial in a random order and were asked to indicate via button press the order in which the objects originally appeared (Fig. 1C). Only nonprobed objects were included to reduce interference from probed objects, which had appeared twice in each trial. As in the coarse temporal memory test, participants were instructed not to respond to a trial unless they remembered all three objects from the previous working memory task.

Temporal recall accuracy. Fine temporal memory scores were calculated for each trial based on the number of pairwise temporal relationships correctly recalled among the three objects. A score of 3 was given when all three objects were recalled in the correct order (e.g., 1,2, 3: 1 before 2,1 before 3,2 before 3 ). A score of 2 was given when two pairs of objects were recalled in the correct order (e.g., 1, 3, 2: 1 before 3, 1 before 2), and a score of 1 was given when only one pair of objects was in the correct order (e.g., 3, 1, 2: 1 before 2). A score of 0 was given when all three objects were placed in reverse order (e.g., 3, 2, 1). For the fMRI analysis, trials with a score of 3 were binned as Hits, and trials with a score of 0 or 1 were binned as Misses, resulting in two groups of approximately equal size (mean Hits = 28.86; mean Misses $=25.50$ ). Data from one participant was excluded from the fine temporal memory analysis because of a technical problem during data collection.

Coarse temporal accuracy was calculated by comparing the participant's estimate of when a trial occurred (i.e., the location of the mark on the horizontal line) with the actual time of occurrence. If the participant marked a point on the line corresponding to the 20th trial, for example, when the trial had in fact been the fifth, then the memory for that trial was considered to be displaced forward in time. Unfortunately, a general bias among participants against using the ends of the scale, as well as a tendency to bias responses toward the beginning of the scale, prevented us from using absolute deviation as a measure of coarse temporal accuracy. This is illustrated in Figure 2A, which depicts the responses of a single representative participant. The participant marked no trials as having occurred during the last sixth of the experiment. If accuracy were measured as the deviation between actual and estimated time of occurrence (i.e., distance from the dashed line), then nearly all the trials from the last third of the experiment would be considered inaccurate.

To account for these response biases, we measured accuracy for each participant by regressing the estimated temporal position against the actual temporal position of each trial (Fig. $2 \mathrm{~A}$, solid line). The residual error associated with a particular trial (i.e., the distance from the regression line) represents the degree to which the participant's memory of the time of occurrence was displaced forward or backward in time. Errors above the regression line indicate forward displacement, errors below the line indicate backward displacement, and small errors indicate greater accuracy relative to larger errors. For the fMRI analyses, the one-third of trials with the smallest absolute errors were binned as Hits (Fig. $2 \mathrm{~A}$, green), and the one-third of trials with the most extreme absolute errors were binned as Misses (Fig. $2 A$, red).

Imaging acquisition and preprocessing. MRI data were acquired using a 3T Siemens Trio scanner equipped with an eight-channel phased-array head coil. Padding and adjustable head restraints were used to minimize head motion. Functional data were obtained with a gradient echoplanar imaging sequence (repetition time, $2 \mathrm{~s}$; echo time, $25 \mathrm{~ms}$; field of view, $220 ; 64 \times 64$ matrix). Each volume consisted of 34 axial slices, each with a slice thickness of $3.4 \mathrm{~mm}$ with no interslice gap, resulting in a voxel size 
of $3.4 \mathrm{~mm}$ isotropic. Coplanar T1-weighted images were acquired using an MP-RAGE sequence (192 slices, $256 \times 256$ matrix, $1 \mathrm{~mm}$ isotropic voxels).

fMRI preprocessing and statistical analyses were conducted using SPM5 (http://www.fil.ion.ucl.ac.uk/spm/) customized with in-house scripts. Functional images were sinc interpolated to correct for slice timing, realigned to the mean image using a six-parameter, rigid-body transformation, and normalized to a common template (Montreal Neuroimaging Institute) using subject-specific dropout masks to reduce image distortion (Brett et al., 2001). Normalized images were resliced into $3 \mathrm{~mm}$ isotropic voxels and smoothed with an $8 \mathrm{~mm}$ full-width half-maximum Gaussian filter.

$f M R I$ data analysis. Two different general linear model (GLM) analyses were conducted on the functional data at the individual level, one to detect activity associated with fine temporal accuracy, the other coarse temporal accuracy. Both contained identical design elements. Separate regressors were included to model each phase of the working memory task - encoding, delay, and probe-with the onsets and offsets of adjacent task phases spaced apart to minimize the chance of activity in one phase loading on the covariate of the next phase. Trials binned as Hits and Misses were modeled separately, and the remaining trials, along with trials associated with incorrect responses on the working memory task, were modeled with a third set of regressors. Task regressors were created by convolving the appropriate boxcar functions with the canonical hemodynamic response function included in SPM5. Additional nuisance regressors were included to model the six movement parameters obtained during realignment, along with regressors modeling baseline and sessions effects.

Contrast images were generated for each participant by subtracting the parameter estimates associated with Miss trials from those associated with Hit trials during each task phase, and these images were submitted to random-effects $t$ tests at the group level. The resulting statistical parametric maps (SPMs) were subjected to an uncorrected voxelwise threshold of $p<0.001$. To correct for multiple comparisons, a cluster extent threshold of six contiguous voxels was adopted within the MTL and PFC regions of interest, corresponding to a corrected $p<0.05$. A more stringent threshold of 12 voxels was used for data outside of these regions. Extent thresholds were calculated based on gray matter volume using a Monte Carlo simulation implemented by the AFNI AlphaSim tool (http://afni.nimh.nih.gov/pub/dist/doc/manual/AlphaSim.pdf).

To improve statistical power within the MTL, contrast images were registered across participants using the Diffeomorphic Demons algorithm included in the MedINRIA software package (v. 1.8.0; Asclepios Research Team) (Vercauteren et al., 2007; Yassa and Stark, 2009). Anatomical regions of interest, including the bilateral hippocampus and parahippocampal gyrus, were manually traced for each participant, using the normalized coplanar MP-RAGE images for definition. A single participant was then chosen as a model, to which the regions of interest (ROIs) of the remaining participants were registered. The resulting deformation fields were applied to the individual contrast maps, resulting in a parallel set of group level SPMs with optimized MTL alignment. Activated clusters within the MTL region of interest were reported from these aligned SPMs, whereas all non-MTL clusters were reported from the original, untransformed SPMs.

To analyze the change in multivoxel activation patterns across trials, we first selected PFC and MTL clusters from the coarse temporal memory contrast that were sufficiently large $(k>30)$. An exception was made for the hippocampus cluster from the coarse memory analysis, which was enlarged to 95 voxels by dropping the activation threshold to $p<0.005$. Vectors of delay period beta values were extracted from each of these regions of interest, one vector for each trial of the experiment. The trialspecific beta maps from which these values were taken were estimated from an additional GLM analysis that modeled each trial separately. Vector similarity was calculated between each trial and its temporally adjacent neighbors (lags $\pm 1,2,3$, and 4 ) by taking the Euclidean distance between the two trial vectors and dividing by the number of voxels in the ROI. These distance indices were calculated separately for trials binned as accurate and inaccurate according to responses on the coarse temporal memory test. The first and last four trials of the experiment were dis-

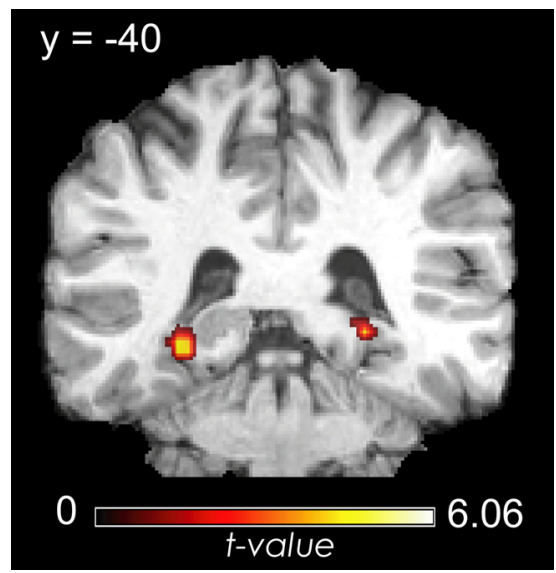

Figure 3. PHC activity predicts fine temporal accuracy. Regions showing greater encoding period activity for trials binned as Hits versus Misses on the fine temporal memory test.

carded from the analysis, since distance information was not available for these trials at all lags.

\section{Results}

\section{Behavioral}

Accuracy on the working memory task was high overall (mean $=$ $97 \%$, SEM $=1 \%$ ), with no participant answering $<89 \%$ of trials correctly. Mean reaction time for correct responses was $1136 \mathrm{~ms}$ $(\mathrm{SEM}=79 \mathrm{~ms})$. Subsequent performance on the fine temporal memory test was significantly above chance, with the average participant able to recall the correct serial order for 35\% of trials (SEM $=2 \%)$, compared with the $17 \%$ expected by random guessing $(p=0.00001)$. In contrast, a low score of 0 or 1 was obtained on only $40 \%$ of trials, less than the $50 \%$ expected by guessing ( $p=0.06)$. The average percentage of trials for which the participant failed to recognize one or more of the objects was $6 \%($ SEM $=2 \%)$.

The correlation between actual and recalled temporal position on the coarse temporal memory test was 0.37 on average $(p=$ 0.001 ), indicating that participants were able to place a given trial in its correct temporal context with a significant degree of accuracy. Figure $2 B$ shows the absolute residual error associated with each trial, group-averaged and plotted against time. It reveals a significant linear relationship $(r=-0.43, p<0.001)$, with accuracy increasing the later in the experiment a trial occurs. Similar examples of a temporal recency effect have been reported previously (Hinrichs and Buschke, 1968; Hintzman et al., 1973; McCormack, 1984). The average percentage of trials for which the participant failed to recognize the object was $2 \%(\mathrm{SEM}=1 \%)$.

\section{Imaging}

Fine temporal memory

To identify regions in which activity during the working memory task predicted accurate performance on the fine temporal memory test, we contrasted trials for which participants recalled the correct serial order (a score of 3 ) with trials for which little or no serial order information was retained (a score of 0 or 1 ). As shown in Figure 3 and Table 1, this contrast revealed significant activation within the posterior parahippocampal cortex during both the encoding and delay periods of the task. No significant activity was observed in other MTL regions or in the prefrontal regions of interest. A whole-brain analysis revealed additional delay period activation in left premotor cortex as well as a number of posterior visual processing areas (Henson et al., 2000). 
Table 1. Fine temporal accuracy

\begin{tabular}{llcll}
\hline & BA & MNI coordinates & $\mathrm{T}(\mathrm{df}=13)$ & Cluster size \\
\hline MTL & & & \\
Encoding & & & & \\
R PHC/Fusiform & 37 & $30,-48,-3$ & 6.06 & 47 \\
L PHC/Fusiform & $19 / 37$ & $-27,-60,-3$ & 5.40 & 54 \\
Delay & & & & 9 \\
R PHC/Fusiform & 30 & $24,-45,-18$ & 4.64 & \\
Whole Brain & & & & 35 \\
Delay & & & & 36 \\
R Calcarine & $19 / 37$ & $27,-51,9$ & 7.11 & 94 \\
L Mid Occipital & $19 / 39$ & $-39,-78,21$ & 6.66 & 14 \\
L Lingual & 18 & $-12,-72,-6$ & 5.58 & \\
L Premotor & 8 & $-24,24,54$ & 5.49 & \\
\hline
\end{tabular}

L, Left; $R$, right.

Table 2. Coarse temporal accuracy

\begin{tabular}{|c|c|c|c|c|}
\hline & $\mathrm{BA}$ & MNI coordinates & $\mathrm{T}(\mathrm{df}=14)$ & $\begin{array}{l}\text { Cluster } \\
\text { size }\end{array}$ \\
\hline \multicolumn{5}{|l|}{ MTL } \\
\hline \multicolumn{5}{|l|}{ Delay } \\
\hline RHPC & 35 & $21,-9,-21$ & 4.85 & 7 \\
\hline \multicolumn{5}{|l|}{ PFC } \\
\hline \multicolumn{5}{|l|}{ Delay } \\
\hline L RLPFC & 10 & $-27,54,18$ & 6.40 & 58 \\
\hline L VLPFC & $44 / 45$ & $-45,18,-6$ & 6.34 & 90 \\
\hline LDLPFC & $9 / 46$ & $-45,36,21$ & 4.28 & 7 \\
\hline \multicolumn{5}{|l|}{ Probe } \\
\hline LSup Frontal & 9 & $-15,51,39$ & 4.89 & 18 \\
\hline \multicolumn{5}{|l|}{ Whole Brain } \\
\hline \multicolumn{5}{|l|}{ Encoding } \\
\hline L Premotor & 6 & $-42,0,21$ & 5.53 & 17 \\
\hline R Angular & 39 & $45,-66,33$ & 4.71 & 19 \\
\hline L Premotor & 6 & $-24,21,45$ & 4.03 & 11 \\
\hline \multicolumn{5}{|l|}{ Delay } \\
\hline R Angular & 40 & $42,-45,30$ & 8.69 & 130 \\
\hline L Premotor/Anterior Cingulate & $6 / 8 / 32 / 24$ & $-45,-3,39$ & 8.22 & 468 \\
\hline R Putamen & & $27,0,0$ & 7.11 & 73 \\
\hline L Mid Temporal & $21 / 22$ & $-51,-21,0$ & 6.21 & 117 \\
\hline L Pallidum/L Putamen & & $-24,-12,3$ & 6.08 & 97 \\
\hline L Cerebellum & & $-45,-66,-24$ & 5.71 & 27 \\
\hline L Angular & 39 & $-33,-54,30$ & 5.54 & 125 \\
\hline L Calcarine & $17 / 18$ & $-9,-99,-6$ & 5.54 & 35 \\
\hline R Premotor & 8 & $51,21,39$ & 5.39 & 37 \\
\hline R Insula & 48 & $48,6,3$ & 5.10 & 51 \\
\hline L Mid Occipital & 19 & $-30,-87,30$ & 5.05 & 23 \\
\hline \multicolumn{5}{|l|}{ Probe } \\
\hline L Mid Temporal & $20 / 21$ & $-66,-21,-15$ & 7.43 & 68 \\
\hline R Inf Temporal & 20 & $60,-30,-21$ & 5.84 & 22 \\
\hline R Sup Frontal & 8 & $21,21,48$ & 5.53 & 30 \\
\hline L Post Cingulate & 23 & $0,-48,33$ & 5.42 & 60 \\
\hline L Premotor & 9 & $-42,21,48$ & 5.40 & 24 \\
\hline R Mid Temporal & 21 & $60,-33,-6$ & 5.09 & 19 \\
\hline
\end{tabular}

L, Left; $R$, right; Sup, superior; Inf, inferior.

\section{Coarse temporal memory}

Table 2 lists regions of significant activation revealed by contrasting accurate with inaccurate trials based on the residual errors calculated from each participant's coarse memory posttest (see Materials and Methods). Temporal context memory was predicted by activity, primarily during the delay period, in a robust network of regions (Fig. $4 A$ ). Within our a priori regions of interest, these included the left rostrolateral PFC (RLPFC; BA 10), left ventrolateral PFC (VLPFC; BA 44/45), and left dorsolateral PFC (DLPFC; BA 45/46), as well as the right anterior hippocam- pus. The whole-brain analysis revealed additional delay-period activation in bilateral premotor cortex, bilateral angular gyrus, and the basal ganglia. A test of the opposite contrast, which would indicate greater activity for inaccurate than for accurate trials, revealed nothing above threshold.

To confirm that the activation described above predicted general temporal accuracy and was not driven primarily by errors in one direction or another, we compared delay period activity for accurate trials to activity for trials with backward and forward displacement errors within each of our PFC and MTL clusters. The line graphs in Figure $4 B$ show that mean activity was greater for trials that were later recalled accurately than for trials that were displaced either backward or forward in time, respectively, in the $\operatorname{RLPFC}\left(F_{(1,14)}=4.96, p=0.04 ; F_{(1,14)}=13.89, p=0.002\right)$, $\operatorname{DLPFC}\left(F_{(1,14)}=3.18, p=0.10 ; F_{(1,14)}=23.13, p<0.001\right)$, $\operatorname{VLPFC}\left(F_{(1,14)}=10.31, p=0.006 ; F_{(1,14)}=11.34, p=0.005\right)$, and hippocampus $\left(F_{(1,14)}=7.11, p=0.003 ; F_{(1,14)}=7.11, p=\right.$ 0.007).

Given that late-occurring trials were, on average, relatively more accurate than earlier trials (Fig. $2 B$ ), we conducted an additional analysis to rule out time of presentation as a possible confound. Including additional regressors in the GLM to model a linear change in response magnitude over time had little effect on the observed pattern of activation. Thus, it is unlikely that our results are due to any time-dependent process, such as fatigue or training effects.

Having identified regions in which the magnitude of activation during encoding predicted accurate coarse temporal memory, we went on to investigate whether the pattern of activation across voxels within those regions might also convey temporal information. Several computational theories of temporal order memory (Brown et al., 2000; Howard and Kahana, 2002; Polyn et al., 2009a) have proposed a gradually evolving contextual representation that, when bound to an item at encoding, provides an important cue as to when that item was encountered. Given that multivoxel pattern analysis has been used successfully to differentiate between cognitive states (Haynes and Rees, 2005; Polyn et al., 2005; Hampton and O'Doherty, 2007; Haynes et al., 2007), we reasoned that if any part of our activated network were involved in maintaining and integrating contextual representations, then the multivoxel patterns in those areas should become gradually more dissimilar with increasing temporal distance (Manns et al., 2007; Manning et al., 2010). We also expected that pattern similarity between a given trial and temporally adjacent trials would relate to performance on the coarse temporal memory test. Previous behavioral work suggests that order memory is improved when items are encoded in distinctive contexts, such as in different rooms (Block, 1982) or during different task blocks (Underwood, 1977). Accordingly, we predicted that the more distinctive a participant's internal representation of context for a given trial, the more accurately the participant would be able to place that trial in time.

Using PFC and MTL clusters from the coarse temporal memory contrast as regions of interest, we first extracted a vector of delay period beta values from each trial of the experiment, with the length of that vector $(\mathrm{N})$ equal to the number of voxels in the ROI. We then measured the pattern similarity between the target trial and adjacent trials at lags $\pm 1,2,3$, and 4 for both accurate and inaccurate trials. A multivariate distance index was created by plotting each vector as a point in $\mathrm{N}$-dimensional space, calculating the Euclidean distance between target and lag trial vectors, and dividing by $\mathrm{N}$ (to compensate for small differences in voxel 
count). Distance indices from the RLPFC ROI are plotted as a function of accuracy and lag in Figure 5A. A 2 (accuracy) $\times 2$ (direction: forward vs backward) $\times 4$ (lag) repeated-measures ANOVA revealed significant main effects for accuracy $\left(F_{(1,14)}=6.59, p=0.02\right)$ and lag $\left(F_{(1,12)}=8.64, p=0.003\right)$ and a marginally significant main effect for direction $\left(F_{(1,14)}=4.30, p=0.06\right)$. There were no significant interaction effects. These findings indicate that activation patterns in the RLPFC were progressively more dissimilar over increasing temporal lags and that the dissimilarity between a given trial and its adjacent neighbors in either direction was greater for accurate than for inaccurate trials. The same analysis conducted on pattern similarity in the VLPFC (Fig. 5B), in contrast, yielded only a main effect of lag $\left(F_{(1,12)}=9.88, p=0.001\right)$. An analysis of the right hippocampus region of interest produced no significant effects.

The effect of accuracy on pattern similarity illustrated in Figure $5 A$ could potentially be discounted as a statistical artifact, given that the RLPFC region of interest was defined functionally as an area in which activity was significantly greater during accurate than during inaccurate trials. Due to regression to the mean, activity in this region during any given accurate trial will, on average, be greater than during adjacent trials, and differences in mean activity alone could conceivably drive differences in pattern similarity. To account for this possibility, we conducted an additional multivariate analysis on the RLPFC data, this time calculating the distance between meancentered pattern vectors to control for differences in overall activity level. Pattern dissimilarity between accurate trials and adjacent trials remained significantly greater than for inaccurate trials $\left(F_{(1,14)}=\right.$ $5.88, p=0.03)$, indicating that the accuracy effect cannot be accounted for by differences in mean activity alone.

\section{Discussion}

In this experiment, we examined the neural correlates of temporal context encoding at two timescales, one associated with the order of events within a single encoding episode, the other with the order of events across encoding episodes. Long-term memory for fine temporal sequences was predicted by activity in the posterior parahippocampal cortex (PHC), whereas coarse temporal memory was predicted by activity in RLPFC, DLPFC, VLPFC, and the hippocampus. Additional multivoxel pattern analyses revealed evidence implicating the RLPFC in the representation of timevarying contextual states in a manner similar to that proposed by computational theories of temporal context memory.

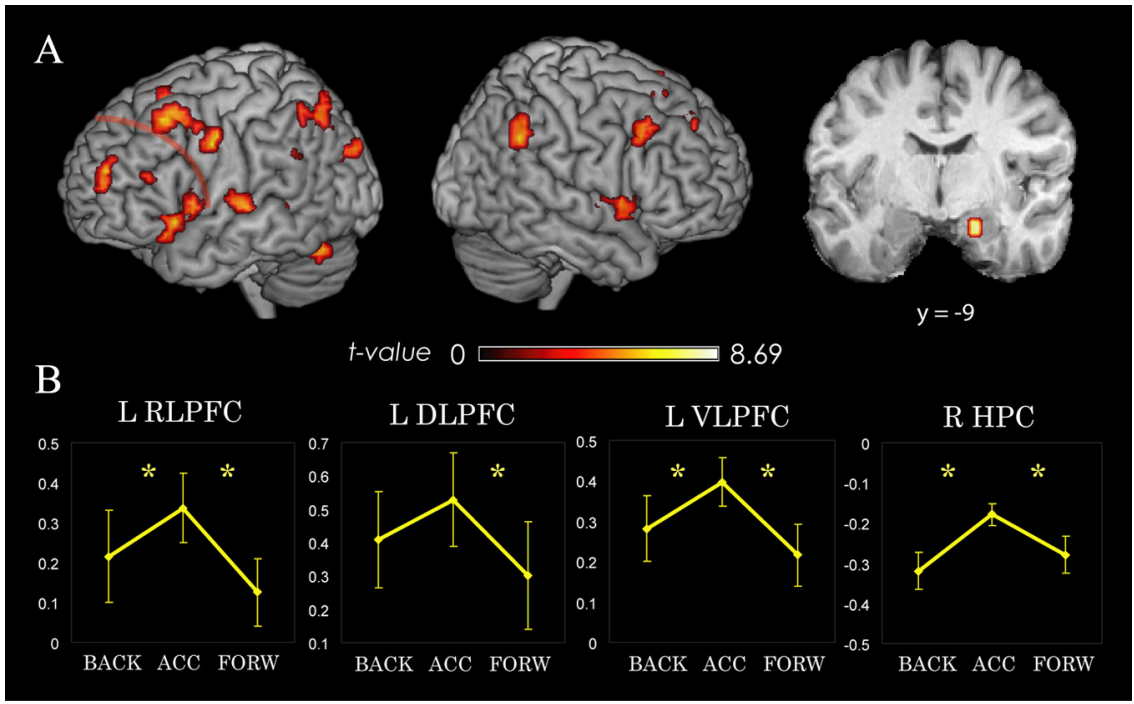

Figure 4. Prefrontal and hippocampal activity predicts coarse temporal accuracy. $\boldsymbol{A}$, Regions showing greater delay period activity for trials binned as Hits versus Misses on the coarse temporal memory test. $\boldsymbol{B}$, Plots show parameter estimates for accurate (ACC), backward displaced (BACK), and forward displaced (FORW) trials. L, Left; R, right. Error bars, \pm 1 SEM. * Significance at $p<0.05$.

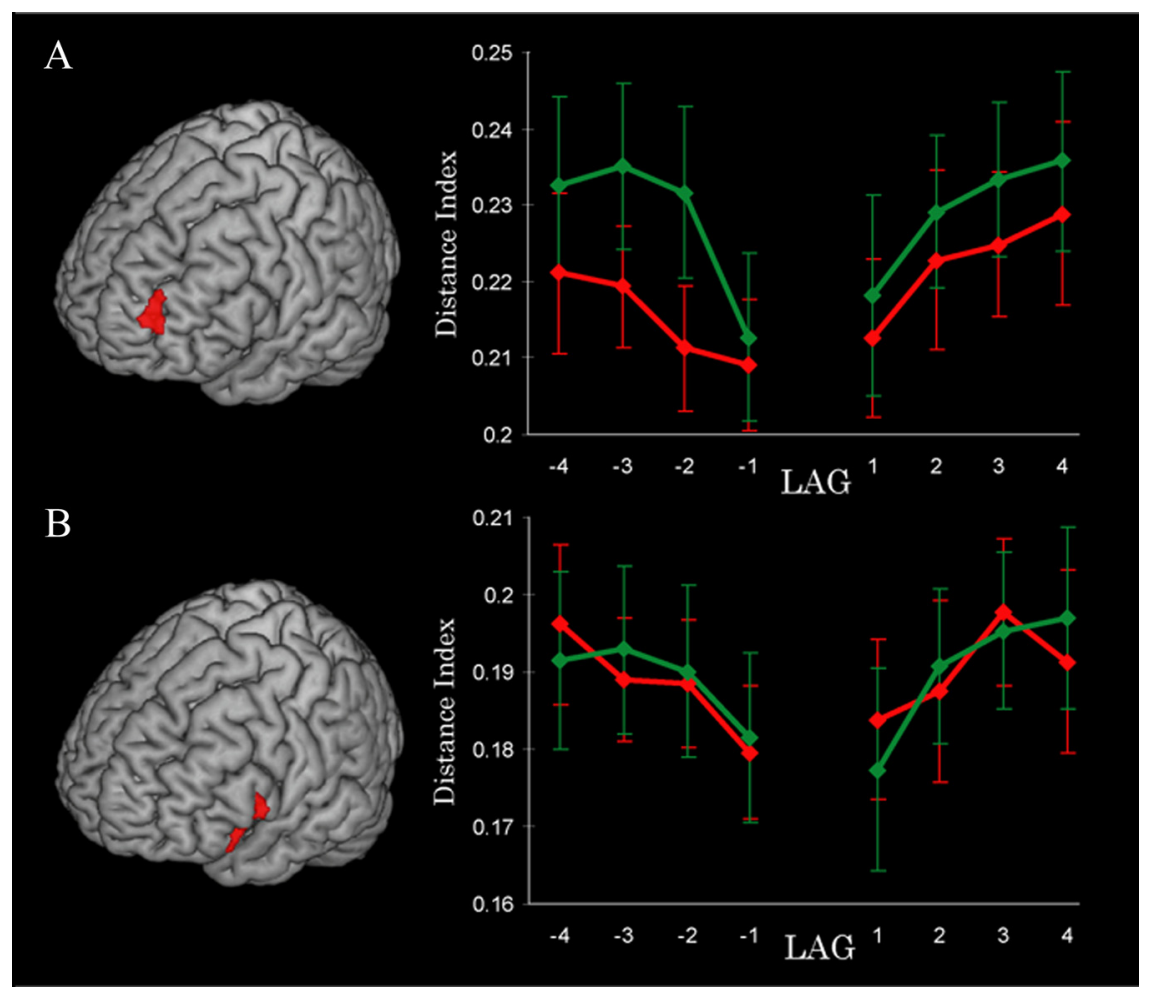

Figure 5. Multivoxel pattern change in $\operatorname{RLPFC}$ predicts coarse temporal accuracy. $\boldsymbol{A}, \boldsymbol{B}$, Within $\operatorname{RLPFC}(\boldsymbol{A})$ and $\operatorname{VLPFC}(\boldsymbol{B})$ regions of interest, multivariate distances between activation patterns associated with target trials and adjacent trials (lags $\pm 1-4$ ) are plotted separately for accurate (green) and inaccurate (red) trials.

\section{Lateral PFC supports coarse temporal context memory}

Findings from human lesion and neuroimaging studies implicating the PFC in long-term temporal order memory have typically used recency discrimination tasks, which require a decision about the relative order of two items on a previously studied list. $\mathrm{Pa}$ tients with PFC lesions often perform poorly at recency discrimination, even though item recognition is relatively spared (McAndrews and Milner, 1991; Milner et al., 1991; Butters et al., 
1994; Kesner et al., 1994; Duarte et al., 2010), and imaging studies have likewise shown increased PFC activation during recency discrimination relative to item recognition tasks (Cabeza et al., 1997, 2002; Suzuki et al., 2002). However, these earlier results could also be interpreted to suggest a more general role for the PFC in making fine-grained retrieval decisions (e.g., by implementing domain general monitoring and evaluative processes) (Ranganath et al., 2000). In contrast, the results of the present study provide direct evidence for lateral PFC involvement in temporal context encoding.

The finding that activity in the PFC predicted coarse temporal memory but not memory for fine temporal sequences might seem to contradict evidence from primate lesion (Petrides, 1991) and neurophysiology studies (Ninokura et al., 2003, 2004), as well as from human neuroimaging studies (Amiez and Petrides, 2007) indicating a role for the PFC in the short-term maintenance of sequences of objects. Our results are consistent in this respect with those of Tubridy and Davachi (2010) (see below), who also did not observe evidence linking PFC encoding activity to subsequent long-term serial order memory. However, both of these fMRI experiments were designed to investigate encoding mechanisms supporting long-term retention rather than the short-term maintenance of sequence information, and further study is needed to understand the relationship between these two processes.

Our results indicate a role for multiple lateral prefrontal regions in encoding the types of information that contribute to successful coarse temporal context judgments. Numerous studies have implicated the VLPFC in the encoding of item information and the DLPFC in encoding the relationships among items (Blumenfeld and Ranganath, 2006, 2007; Murray and Ranganath, 2007). Since both item and relational information likely support coarse temporal memory, the effects in VLPFC and DLPFC fit well with these findings. However, the effects in RLPFC are relatively novel and indicate a potentially important role for this region in encoding temporal context.

Although the RLPFC was one of several regions showing increased activity during trials associated with successful coarse temporal memory judgments, changes in the spatial pattern of RLPFC activity also conveyed information about temporal context. Multivoxel patterns in both the RLPFC and VLPFC changed gradually with time, becoming increasingly dissimilar the greater the temporal distance between trials (Fig. 5). However, only in the RLPFC did trial-to-trial pattern changes during encoding predict subsequent coarse temporal accuracy. The temporal distinctiveness of activation patterns in the RLPFC (i.e., the difference in the activation pattern between the current trial and temporally adjacent trials) was greater on trials that were associated with accurate coarse memory judgments, and this was true even when controlling for differences in activation magnitude. These results suggest that the RLPFC may support the kinds of contextual representations that have been proposed in computational models of temporal context memory (Polyn and Kahana, 2008).

We have proposed previously that the RLPFC may be important in selecting and inhibiting cognitive sets, the high-level rule representations that specify the items and relationships relevant within a given behavioral context (Braver et al., 2003; Bunge et al., 2005; Dosenbach et al., 2007; Ranganath and Blumenfeld, 2008; Sakai, 2008). Cognitive sets are likely to integrate many sources of information useful in specifying behavioral context; for example, goals, sensory information, and information on internal states. We speculate that by continuously updating this integrated rep- resentation, the RLPFC may help to segment ongoing experience into discrete episodes (Zacks et al., 2007). A sudden shift in cognitive set would be expected to lead to a more distinctive contextual representation (Polyn et al., 2009b), which may in turn facilitate memory for when the event took place (Underwood, 1977; Block, 1982). This would explain why, in the RLPFC, trials associated with accurate coarse temporal memory are characterized by a more pronounced jump in the ongoing multivoxel pattern drift. However, this explanation is highly speculative, and future studies will be necessary to test the role we propose for RLPFC in temporal context encoding.

\section{Hippocampus and PHC support temporal context memory}

Results from lesion and physiology studies in rats suggest that the hippocampus plays a critical role in encoding temporal context information. For example, hippocampal lesions have been shown to impair memory for the order of a series of odors (Fortin and Eichenbaum, 2002; Kesner et al., 2002), and activity in ensembles of hippocampal neurons during the encoding of odor sequences has been shown to predict subsequent order memory (Manns et al., 2007). The results of the present study, along with a recent study by Tubridy and Davachi (2010), are the first to our knowledge linking activity in the human hippocampus and PHC with the successful encoding of temporal context. Tubridy and Davachi (2010) scanned participants as they encoded serially presented word triplets and tested for triplet order memory at the end of each scanning run. They reported results very similar to our own fine temporal memory contrast, with activity in both the $\mathrm{PHC}$ and hippocampus that predicted triplet order memory. Although we did not find significant hippocampal activity in the fine temporal memory contrast, this could be due to a number of methodological differences between the two studies (e.g., stimulus material, study test lag, etc.).

Human neuroimaging studies have also reported activity in both the hippocampus and PHC associated with encoding other nontemporal forms of contextual information (Davachi et al., 2003; Ranganath et al., 2004; Kensinger and Schacter, 2006; Kirwan et al., 2008; Ross and Slotnick, 2008). Based on these and other results, several researchers have suggested that the PHC might contribute to episodic memory formation by representing the spatiotemporal and semantic context in which an item was encountered (Bar and Aminoff, 2003; Davachi, 2006; Diana et al., 2007; Eichenbaum et al., 2007; Ranganath, 2010) and that the hippocampus serves to encode bound representations of item in context (Davachi, 2006; Diana et al., 2007; Eichenbaum et al., 2007; Konkel and Cohen, 2009).

One might argue that the hippocampus and $\mathrm{PHC}$ encode both general familiarity and contextual associations, and that the results of the present study simply reflect a disproportionate increase in activity during the encoding of strong versus weak memories (Squire et al., 2007). Indeed, memory strength can provide an important temporal cue, in that recent memories tend to be stronger than more remote memories (Hinrichs, 1970). According to this interpretation, we would expect to see hippocampal activity in the coarse temporal memory analysis that is greater for trials that were judged to have occurred more recently than they actually did. However, as illustrated in Figure $4 B$, activity during trials that showed a forward bias did not differ significantly from activity during trials that were biased in the opposite direction, whereas activity during accurate trials was significantly greater than during either forward- or backwardbiased trials. This suggests that activity in the hippocampus reflected the encoding of contextual information supporting 
accurate temporal memory but was insensitive to familiarity or overall memory strength (Cohn et al., 2009; Diana et al., 2010).

\section{Conclusion}

The results of this study provide insight into the manner in which temporal information is encoded in the human brain and suggest the possibility that complementary encoding mechanisms may support temporal memory at different timescales. The study also demonstrates the feasibility of using multivoxel pattern analysis to study the temporal distinctiveness of neural representations, a methodological approach that could be adapted to address a number of questions about the neural representation of context.

\section{References}

Agster KL, Fortin NJ, Eichenbaum H (2002) The hippocampus and disambiguation of overlapping sequences. J Neurosci 22:5760-5768.

Amiez C, Petrides M (2007) Selective involvement of the mid-dorsolateral prefrontal cortex in the coding of the serial order of visual stimuli in working memory. Proc Natl Acad Sci U S A 104:13786-13791.

Bar M, Aminoff E (2003) Cortical analysis of visual context. Neuron 38:347-358

Block RA (1982) Temporal judgments and contextual change. J Exp Psychol Learn Mem Cogn 8:530-544.

Blumenfeld RS, Ranganath C (2006) Dorsolateral prefrontal cortex promotes long-term memory formation through its role in working memory organization. J Neurosci 26:916-925.

Blumenfeld RS, Ranganath C (2007) Prefrontal cortex and long-term memory encoding: an integrative review of findings from neuropsychology and neuroimaging. Neuroscientist 13:280-291.

Braver TS, Reynolds JR, Donaldson DI (2003) Neural mechanisms of transient and sustained cognitive control during task switching. Neuron 39:713-726.

Brett M, Leff AP, Rorden C, Ashburner J (2001) Spatial normalization of brain images with focal lesions using cost function masking. Neuroimage 14:486-500.

Brown GD, Preece T, Hulme C (2000) Oscillator-based memory for serial order. Psychol Rev 107:127-181.

Bunge SA, Wallis JD, Parker A, Brass M, Crone EA, Hoshi E, Sakai K (2005) Neural circuitry underlying rule use in humans and nonhuman primates. J Neurosci 25:10347-10350.

Butters MA, Kaszniak AW, Glisky EL, Eslinger PJ, Schacter DL (1994) Recency discrimination deficits in frontal lobe patients. Neuropsychology 8:343-353.

Cabeza R, Mangels J, Nyberg L, Habib R, Houle S, McIntosh AR, Tulving E (1997) Brain regions differentially involved in remembering what and when: a PET study. Neuron 19:863-870.

Cabeza R, Anderson ND, Houle S, Mangels JA, Nyberg L (2000) Age-related differences in neural activity during item and temporal-order memory retrieval: a positron emission tomography study. J Cogn Neurosci 12:197-206

Cohn M, Moscovitch M, Lahat A, McAndrews MP (2009) Recollection versus strength as the primary determinant of hippocampal engagement at retrieval. Proc Natl Acad Sci U S A 106:22451-22455.

Davachi L (2006) Item, context and relational episodic encoding in humans. Curr Opin Neurobiol 16:693-700.

Davachi L, Mitchell JP, Wagner AD (2003) Multiple routes to memory: distinct medial temporal lobe processes build item and source memories. Proc Natl Acad Sci U S A 100:2157-2162.

Diana RA, Yonelinas AP, Ranganath C (2007) Imaging recollection and familiarity in the medial temporal lobe: a three-component model. Trends Cogn Sci 11:379-386.

Diana RA, Yonelinas AP, Ranganath C (2010) Medial temporal lobe activity during source retrieval reflects information type, not memory strength. J Cogn Neurosci 22:1808-1818.

Dobbins IG, Rice HJ, Wagner AD, Schacter DL (2003) Memory orientation and success: separable neurocognitive components underlying episodic recognition. Neuropsychologia 41:318-333.

Dosenbach NU, Fair DA, Miezin FM, Cohen AL, Wenger KK, Dosenbach RA, Fox MD, Snyder AZ, Vincent JL, Raichle ME, Schlaggar BL, Petersen SE (2007) Distinct brain networks for adaptive and stable task control in humans. Proc Natl Acad Sci U S A 104:11073-11078.
Duarte A, Henson RN, Knight RT, Emery T, Graham KS (2010) Orbitofrontal cortex is necessary for temporal context memory. J Cogn Neurosci 22:1819-1831.

Eichenbaum H, Yonelinas AP, Ranganath C (2007) The medial temporal lobe and recognition memory. Annu Rev Neurosci 30:123-152.

Eyler-Zorilla LT, Aguirre GK, Zarahn E, Cannon TD, D’Esposito M (1996) Activation of the prefrontal cortex during judgments of recency: a functional MRI study. Neuroreport 7:2803-2806.

Fortin NJ, Agster KL, Eichenbaum HB (2002) Critical role of the hippocampus in memory for sequences of events. Nat Neurosci 5:458-462.

Friedman WJ (1993) Memory for the time of past events. Psychol Bull 113:44-66.

Hampton AN, O'Doherty JP (2007) Decoding the neural substrates of reward-related decision making with functional MRI. Proc Natl Acad Sci U S A 104:1377-1382.

Haynes JD, Rees G (2005) Predicting the orientation of invisible stimuli from activity in human primary visual cortex. Nat Neurosci 8:686-691.

Haynes JD, Sakai K, Rees G, Gilbert S, Frith C, Passingham RE (2007) Reading hidden intentions in the human brain. Curr Biol 17:323-328.

Henson RN, Burgess N, Frith CD (2000) Recoding, storage, rehearsal, and grouping in verbal short-term memory: an fMRI study. Neuropsychologia 38:426-440.

Hinrichs JV (1970) A two-process memory-strength theory for judgment of recency. Psychol Rev 77:223-233.

Hinrichs JV, Buschke H (1968) Judgment of recency under steady-state conditions. J Exp Psychol 78:574-579.

Hintzman DL, Block RA, Summers JJ (1973) Contextual associations and memory for serial position. J Exp Psychol 97:220-229.

Howard MW, Kahana MJ (2002) A distributed representation of temporal context. J Math Psychol 46:269-299.

Kensinger EA, Schacter DL (2006) Amygdala activity is associated with the successful encoding of item, but not source, information for positive and negative stimuli. J Neurosci 26:2564-2570.

Kesner RP, Novak JM (1982) Serial position curve in rats: role of the dorsal hippocampus. Science 218:173-175.

Kesner RP, Hopkins RO, Fineman B (1994) Item and order dissociation in humans with prefrontal cortex damage. Neuropsychologia 32:881-891.

Kesner RP, Gilbert PE, Barua LA (2002) The role of the hippocampus in memory for the temporal order of a sequence of odors. Behav Neurosci 116:286-290

Kimura HM, Hirose S, Kunimatsu A, Chikazoe J, Jimura K, Watanabe T, Abe O, Ohtomo K, Miyashita Y, Konishi S (2010) Differential temporoparietal cortical networks that support relational and item-based recency judgments. Neuroimage 49:3474-3480.

Kirwan CB, Wixted JT, Squire LR (2008) Activity in the medial temporal lobe predicts memory strength, whereas activity in the prefrontal cortex predicts recollection. J Neurosci 28:10541-10548.

Konishi S, Uchida I, Okuaki T, Machida T, Shirouzu I, Miyashita Y (2002) Neural correlates of recency judgment. J Neurosci 22:9549-9555.

Konkel A, Cohen NJ (2009) Relational memory and the hippocampus: representations and methods. Front Neurosci 3:166-174.

Lehn H, Steffenach HA, van Strien NM, Veltman DJ, Witter MP, Håberg AK (2009) A specific role of the human hippocampus in recall of temporal sequences. J Neurosci 29:3475-3484.

Mangels JA (1997) Strategic processing and memory for temporal order in patients with frontal lobe lesions. Neuropsychology 11:207-221.

Manning JR, Polyn SM, Baltuch G, Litt B, Kahana MJ (2010) Oscillatory patterns in temporal lobe reveal context reinstatement during memory search. Proc Natl Acad Sci U S A, in press.

Manns JR, Howard MW, Eichenbaum H (2007) Gradual changes in hippocampal activity support remembering the order of events. Neuron 56:530-540.

Marshuetz C (2005) Order information in working memory: an integrative review of evidence from brain and behavior. Psychol Bull 131:323-339.

McAndrews MP, Milner B (1991) The frontal cortex and memory for temporal order. Neuropsychologia 29:849-859.

McCormack PD (1984) Temporal coding by young and elderly adults in a list-discrimination setting. Bull Psychon Soc 22:401-402.

Milner B (1971) Interhemispheric differences in the localization of psychological processes in man. Br Med Bull 27:272-277.

Milner B, Petrides M, Smith ML (1985) Frontal lobes and the temporal organization of memory. Hum Neurobiol 4:137-142. 
Milner B, Corsi P, Leonard G (1991) Frontal-lobe contribution to recency judgments. Neuropsychologia 29:601-618.

Murray LJ, Ranganath C (2007) The dorsolateral prefrontal cortex contributes to successful relational memory encoding. J Neurosci 27:5515-5522.

Ninokura Y, Mushiake H, Tanji J (2003) Representation of the temporal order of visual objects in the primate lateral prefrontal cortex. J Neurophysiol 89:2868-2873.

Ninokura Y, Mushiake H, Tanji J (2004) Integration of temporal order and object information in the monkey lateral prefrontal cortex. J Neurophysiol 91:555-560.

Petrides M (1991) Functional specialization within the dorsolateral frontal cortex for serial order memory. Proc Biol Sci 246:299-306.

Polyn SM, Kahana MJ (2008) Memory search and the neural representation of context. Trends Cogn Sci 12:24-30.

Polyn SM, Natu VS, Cohen JD, Norman KA (2005) Category-specific cortical activity precedes retrieval during memory search. Science 310:1963-1966.

Polyn SM, Norman KA, Kahana MJ (2009a) A context maintenance and retrieval model of organizational processes in free recall. Psychol Rev 116:129-156.

Polyn SM, Norman KA, Kahana MJ (2009b) Task context and organization in free recall. Neuropsychologia 47:2158-2163.

Ranganath C (2010) A unified framework for the functional organization of the medial temporal lobes and the phenomenology of episodic memory. Hippocampus 20:1263-1290.

Ranganath C, Johnson MK, D’Esposito M (2000) Left anterior prefrontal activation increases with demands to recall specific perceptual information. J Neurosci 20:RC108.

Ranganath C, Yonelinas AP, Cohen MX, Dy CJ, Tom SM, D'Esposito M (2004) Dissociable correlates of recollection and familiarity within the medial temporal lobes. Neuropsychologia 42:2-13.

Ranganath C, Blumenfeld RS (2008) Prefrontal cortex and memory. In Learning and memory: a comprehensive reference (Eichenbaum H, Byrne JH, eds.), pp. 261-279. London: Academic.
Ross RS, Slotnick SD (2008) The hippocampus is preferentially associated with memory for spatial context. J Cogn Neurosci 20:432-446.

Sakai K (2008) Task set and prefrontal cortex. Annu Rev Neurosci 31:219-245.

Shimamura AP, Janowsky JS, Squire LR (1990) Memory for the temporal order of events in patients with frontal lobe lesions and amnesic patients. Neuropsychologia 28:803-813.

Squire LR, Wixted JT, Clark RE (2007) Recognition memory and the medial temporal lobe: a new perspective. Nat Rev Neurosci 8:872-883.

St. Jacques P, Rubin DC, LaBar KS, Cabeza R (2008) The short and long of it: Neural correlates of temporal-order memory for autobiographical events. J Cogn Neurosci 20:1327-1341.

Suzuki M, Fujii T, Tsukiura T, Okuda J, Umetsu A, Nagasaka T, Mugikura S, Yanagawa I, Takahashi S, Yamadori A (2002) Neural basis of temporal context memory: a functional MRI study. Neuroimage 17: $1790-1796$.

Tubridy S, Davachi L (2010) Medial temporal lobe contributions to episodic sequence encoding. Cereb Cortex. Advance online publication. Retrieved August 10, 2010. doi:10.1093/cercor/bhq092.

Tulving E (1972) Episodic and semantic memory. In Organization and memory (Tulving E, Donaldson W, eds.) New York: Academic.

Tulving E (1993) What is episodic memory? Curr Direct Psychol Sci 2:67-70.

Underwood BJ (1977) Temporal codes for memories: issues and problems. Hillsdale, NJ: Erlbaum.

Vercauteren T, Pennec X, Perchant A, Ayache N (2007) Diffeomorphic demons using ITK's finite difference solver hierarchy. Insight J, 2007 MICCAI Open Science Workshop.

Yassa MA, Stark CE (2009) A quantitative evaluation of cross-participant registration techniques for MRI studies of the medial temporal lobe. Neuroimage 44:319-327.

Zacks JM, Speer NK, Swallow KM, Braver TS, Reynolds JR (2007) Event perception: a mind-brain perspective. Psychol Bull 133:273-293. 